\title{
Autoestima, autoimagem, qualidade de vida e de saúde de mulheres na pós-menopausa
}

\author{
Postmenopausal women self-esteem, self-image, and quality of life and \\ health
}

Autoestima, autoimagen, calidad de vida y salud de las mujeres posmenopáusicas

Kelyn Tedesco ${ }^{1}$, Michele Marinho da Silveira ${ }^{2}$

1 Graduação em Psicologia pela Faculdade Meridional - IMED (Passo Fundo - Rio Grande do Sul, Brasil)

2 Doutora em Gerontologia Biomédica pela Faculdade Meridional - IMED (Rio Grande do Sul, Brasil)

\section{RESUMO}

Este estudo avaliou a autoestima, autoimagem, qualidade de vida e de saúde de mulheres no período de pós-menopausa. Trata-se de uma pesquisa quantitativa, transversal e descritiva, com amostra de conveniência de cinquenta mulheres. Como instrumentos, foram utilizados um questionário socioeconômico, a escala de qualidade de vida (WHOQOL-Bref), questionário de saúde mental da mulher (QSM), questionário de autoestima e autoimagem e escala de avaliação da menopausa (MRS). Observou-se que as mulheres estavam insatisfeitas com sua autoimagem e autoestima, na avaliação da menopausa foram encontrados sintomas moderados,

Autor de Correspondência:

*Michele Marinho da Silveira. E-mail: michele.msilveira@gmail.com 
no questionário de saúde mental o escore foi considerado médio e nos domínios de qualidade de vida teve maior satisfação no físico e menor no meio ambiente. Concluiu-se que as avaliadas apresentaram sintomas considerados moderados da menopausa e não estavam muito satisfeitas com sua autoestima e autoimagem e com o domínio meio ambiente de qualidade de vida.

Palavras-chave: Pós-menopausa. Qualidade de Vida. Autoimagem.

\section{ABSTRACT}

This study evaluated the self-esteem, self-image, quality of life and health of postmenopausal women. This is a quantitative, cross-sectional, and descriptive study, with a convenience sample of fifty women. The instruments used were a socioeconomic questionnaire, the quality of life scale (WHOQOL-Bref), a women's mental health questionnaire (WMHQ), a self-esteem and self-image questionnaire, and a menopause assessment scale (MRS). It was observed that women were dissatisfied with their self-image and self-esteem; in the menopause assessment, moderate symptoms were found, in the mental health questionnaire the score was considered medium, and in the quality of life domains they had greater satisfaction in the physical domain and lower in the environment one. It was concluded that the evaluated women presented moderate symptoms of menopause and were not very satisfied with their self-esteem and selfimage and with the environment domain of quality of life.

Keywords: Postmenopause. Quality of Life. Self Concept.

\section{RESUMEN}

Este estudio evaluó la autoestima, la autoimagen, la calidad de vida y la salud de mujeres posmenopáusicas. Se trata de una investigación cuantitativa, transversal y descriptiva con cincuenta mujeres. Se aplicó un cuestionario socioeconómico, escala de calidad de vida (WHOQOL-Bref), cuestionario de salud mental de la mujer (QSM), cuestionario de autoestima y autoimagen y escala de evaluación de la menopausia (MRS). Se observó que las mujeres estaban descontentas con su autoimagen y autoestima, en la evaluación de la menopausia se encontraron síntomas moderados, en el cuestionario de salud mental se consideró puntaje medio y en los dominios de calidad de vida tuvieron mayor satisfacción en la parte física y más bajo en el medio ambiente. Se concluyó que las evaluadas presentaban síntomas moderados de menopausia y no estaban muy satisfechas con su autoestima e imagen de sí mismas y con el dominio ambiental de la calidad de vida.

Palabras clave: Posmenopausia. Calidad de Vida. Autoimagem. 


\section{INTRODUÇÃO}

Com o processo de envelhecimento humano buscase mais qualidade de vida para as pessoas idosas por meio do conhecimento tecnológico e científico. O essencial é encarar a velhice como um desafio dinâmico, saudável e benéfico, apesar de haver diferenças ao longo das etapas da vida nos aspectos biológicos, individuais, coletivos e culturais ${ }^{1}$.

Segundo dados da PNAD Contínua (Pesquisa Nacional por Amostra de Domicílios Contínua) de 2019, o número de mulheres no Brasil é superior ao de homens. A população brasileira é composta por $48,2 \%$ de homens e $51,8 \%$ de mulheres ${ }^{2}$. Em meio ao ciclo do envelhecimento da população, observase a feminilização da velhice, que representa a predominância das mulheres no público idoso 3 .

Existem diferenças entre o envelhecer masculino e feminino. As mulheres vivenciam a menopausa, que marca o início de uma etapa do ciclo de vida, correspondendo ao último ciclo menstrual. $\mathrm{O}$ climatério é definido como uma fase biológica da vida e não um processo patológico, que compreende a transição entre o período reprodutivo e o não reprodutivo da vida da mulher ${ }^{4}$. O limite etário para o climatério está entre 40 e 65 anos de idade e é dividido em: pré-menopausa que, geralmente, se inicia após os 40 anos, com diminuição da fertilidade em mulheres com ciclos menstruais regulares ou com padrão menstrual similar ao ocorrido durante a vida reprodutiva; perimenopausa que se inicia dois anos antes da última menstruação e vai até um ano após (com ciclos menstruais irregulares e alterações endócrinas); pós-menopausa que começa um ano após o último período menstrual ${ }^{5}$.

As modificações corporais que ocorrem no processo de envelhecimento ocasionam no sujeito uma alteração de sua autoimagem e, por vezes, ocasionam uma distinção da imagem pretendida e a imagem existente. O modo como as pessoas veem sua imagem corporal, têm efeitos de grande relevância em sua qualidade de vida. No decorrer do ciclo vital, as mulheres, provavelmente, movidas por razões culturais, apresentam maior interesse, aflição e desagrado com a autoimagem em contrapartida ao sexo masculino ${ }^{6}$. Isso, pode estar relacionado aos sintomas ocorridos na menopausa como diminuição da libido, aumento da gordura abdominal, presença de calorões, distúrbios do sono e alterações de humor. Logo, na fase climatérica e, por conseguinte pós-menopausa, estes sintomas são definitivos, como ressecamento e sangramento vaginal, alterações hormonais, ganho de peso, distúrbios ósseos e dilatação da região abdominal ${ }^{5}$. Em vista disso, este estudo buscou avaliar a autoestima, autoimagem, qualidade de vida e de saúde de mulheres na pósmenopausa.

\section{MÉTODO}

Trata-se de um estudo quantitativo de cunho transversal e descritivo com amostra não probabilística de conveniência com cinquenta mulheres no período da pós-menopausa, acima de cinquenta anos, residentes nos territórios de abrangência das Unidades Básicas de Saúde de um município da região Norte do Rio Grande do Sul, Brasil. Como critérios de exclusão, mulheres que não tinham capacidade de comunicação verbal, de compreensão do termo de consentimento livre e esclarecido e que não aceitaram participar, destas, seis foram excluídas.

Como instrumentos, foram utilizados um questionário socioeconômico e de saúde criado pelas pesquisadoras especificamente para este estudo, com doze questões fechadas sobre idade, renda familiar, estado civil, ocupação, escolaridade (anos de estudo), moradia, com quem mora, uso do tabaco e álcool; e, quatro questões abertas sobre pós-menopausa, uso de reposição hormonal, se apresenta algum problema de saúde e uso de medicamentos. Também a escala 
WHOQOL-Bref que avalia a percepção da qualidade de vida e foi validada para o português ${ }^{7}$. É composta por 26 questões, duas gerais sobre qualidade de vida e 24 sobre as quatro competências (física, psicológica, relações sociais e meio ambiente). Não possui um ponto de corte, assim quanto mais alto seu escore, melhor é a qualidade de vida do participante, escore variando de 5 a 100 pontos $^{7}$.

A escala de autoestima e autoimagem validada por Stobäus ${ }^{8}$, que avalia os níveis de autoimagem e autoestima, é formada por cinquenta questões que se distribuem em quatro esferas (orgânica, intelectual, emocional e bem-estar social). O escore do questionário pode variar entre 50 e 250 pontos, assim, quanto mais perto de 250 , maior é a autoestima e autoimagem do indivíduo. Além disso, a escala de Avaliação da Menopausa (Menopause Rating Scale - MRS), que calcula os sintomas da menopausa, oficialmente validado e traduzido para o português ${ }^{9}$, pode ser utilizado em qualquer fase da mulher a partir da menopausa. É composta por onze elementos que medem as manifestações climatéricas, divididos por 3 subescalas: (a) Somática (problemas de sono, falta de ar, calores, suores, mal-estar do coração, problemas musculares e nas articulações); (b) Psicológica (ansiedade, irritabilidade, ânimo depressivo e esgotamento físico e mental); e (c) Urogenital (ressecamento vaginal, problemas sexuais e problemas de bexiga). Cada item pode ser pontuado de 0 a 4 , sendo $0=$ nenhum, $1=$ pouco severo, $2=$ moderado, $3=$ severo, $4=$ muito severo. A contagem da escala total é feita pelo total da soma das três subescalas e os sintomas podem ser especificados em: assintomáticos ou escassos (0-4 pontos), leves (5-8 pontos), moderados (9-15 pontos) ou severos (mais de 16 pontos).

E, por fim, o questionário da Saúde Mental da Mulher que foi desenvolvido para analisar os sintomas físicos e mentais experimentados por mulheres durante a pós-menopausa, validado para o português ${ }^{10}$. O questionário de saúde mental da mulher (QSM) possui 37 questões disponibilizando quatro opções de respostas. As perguntas estão separadas em sete etapas, divididas de forma aleatória, que classificam: depressão (questões $3 ; 5 ; 7 ; 8 ; 10 ; 12 ; 25$ ); sintomas somáticos (14;15;16;18;23; 30;35); memória/ concentração $(20 ; 33 ; 36)$; sintomas vasomotores (19; 27); ansiedade/temores (2; 4; 6; 9); comportamento sexual $(24 ; 31 ; 34)$; problemas de sono $(1 ; 11 ; 29)$; sintomas menstruais $(17 ; 22 ; 26 ; 28)$ e atratividade $(13$; 21; 32). O escore de 0-36 é classificado como muito baixo, 37-72 baixo, 73-108 médio, 109-144 bom/ excelente.

Como procedimentos, inicialmente, foi solicitada a autorização na Secretaria de Saúde do município da região Norte do Rio Grande do Sul, Brasil, para realização da pesquisa por intermédio das Unidades Básicas de Saúde. Depois, o projeto de pesquisa foi encaminhado ao Comitê de Ética e Pesquisa da Faculdade Meridional - IMED. Posteriormente à sua aprovação pelo CEP (CAAE: 14625819.7.0000.5319 e parecer: 3.377.013), foi iniciada a pesquisa. Por intermédio de Unidades Básicas de Saúde desse município, realizou-se a avaliação de mulheres no período de pós-menopausa. As Unidades Básicas de Saúde repassaram uma lista com a localização de mulheres com idade entre 50 e 70 anos, residentes em seu território de abrangência para que a pesquisadora fosse até o domicílio realizar a avaliação. A aplicação dos questionários foi realizada no domicílio da própria mulher, que afirmou estar no período de pósmenopausa, tomando todos os cuidados em relação à privacidade da participante e solicitando que a avaliação fosse individual, realizada em um local da casa em que poderia ficar à vontade para responder os questionários/escalas sem interferência de outras pessoas.

Inicialmente, ao chegar ao seu domicílio, era explicada a pesquisa e cada mulher que quisesse participar preenchia o Termo de Consentimento Livre e Esclarecido e respondia sobre informações socioeconômicas e de saúde. Após, eram aplicadas as Escalas de Autoestima e Autoimagem, Qualidade de Vida, Questionário de saúde mental da mulher e Avaliação da Menopausa. 
Para analisar os dados, utilizou-se o programa SPSS 23.0 para Windows. As variáveis foram descritas como média \pm desvio padrão, frequência absoluta e relativa. O teste para normalidade KolmogorovSmirnov foi aplicado para verificar a distribuição dos dados. Para correlacionar as variáveis, utilizou-se o teste de correlação de Spearman. Testes com valor de probabilidade $<0,05$ foram considerados como estatisticamente significativos.

\section{RESULTADOS}

A amostra foi de 50 mulheres com uma média de idade de 57,8 $\pm 5,3$ anos. Quanto aos dados referentes à escolaridade, a média foi 9,4 $\pm 3,2$ anos de estudo em que a frequência escolar varia de 0 a 18 anos. As patologias crônicas não transmissíveis autorreferidas pelas mulheres foram hipertensão arterial com 17 (34\%), diabetes mellitus 7 (14,0\%), insuficiência cardíaca 5 (10,0\%), doenças pulmonares crônicas 4 $(8,0 \%)$ e $2(4,0 \%)$ já tiveram câncer. Das participantes $5(10,0 \%)$ eram fumantes, $10(20,0 \%)$ já fumaram, 11 (22\%) consumiam bebida alcoólica com uma média de $0,60 \pm 1,82$ vezes por semana. Na Tabela 1 observase o perfil socioeconômico e de saúde das mulheres.

A média de idade ao entrar na menopausa foi de $47,16 \pm 4,95$ anos. O número de filhos por mulher obteve uma média de 1,92 $\pm 1,02$, sendo 1,48 $\pm 1,29$ por parto normal, e 0,44 $\pm 0,73$ por cesariana. $\mathrm{Na}$ escala de autoestima e autoimagem não houve uma pontuação elevada, a média foi 131,60 de um total de 250, demonstrando que as mulheres não estão satisfeitas com sua autoimagem e autoestima. $\mathrm{Na}$ avaliação da menopausa, observou-se que o escore foi de 12,18 classificando-se com presença de sintomas moderados e nas suas subescalas, a de sintomas somáticos apresentou sintomas leves, a de psicológicos foram sintomas moderados e a de sintomas urogenitais foram moderados. No questionário de saúde mental da mulher pontuaram 96,74 pontos, classificando os sintomas físicos e
Tabela 1 - Perfil Socioeconômico e de saúde das mulheres na pós-menopausa.

\begin{tabular}{|c|c|c|}
\hline & $\begin{array}{c}\text { Frequência } \\
\qquad \mathbf{N}\end{array}$ & $\begin{array}{c}\text { Percentual } \\
\%\end{array}$ \\
\hline \multicolumn{3}{|l|}{ Estado Civil } \\
\hline Solteira & 4 & $8,0 \%$ \\
\hline $\begin{array}{l}\text { Casada ou com } \\
\text { companheiro }\end{array}$ & 31 & $62,0 \%$ \\
\hline Separada/Divorciada & 9 & $18,0 \%$ \\
\hline Viúva & 6 & $12,0 \%$ \\
\hline \multicolumn{3}{|l|}{ Renda mensal } \\
\hline 1 a 2 salários mínimos & 21 & $42,0 \%$ \\
\hline 3 a 4 salários mínimos & 21 & $42,0 \%$ \\
\hline $\begin{array}{l}\text { Acima de } 5 \text { salários } \\
\text { mínimos }\end{array}$ & 8 & $16,0 \%$ \\
\hline \multicolumn{3}{|l|}{ Ocupação } \\
\hline Aposentada & 14 & $28,0 \%$ \\
\hline Dona de casa & 14 & $28,0 \%$ \\
\hline Trabalhando & 21 & $42,0 \%$ \\
\hline Outro & 1 & $2,0 \%$ \\
\hline \multicolumn{3}{|l|}{ Moradia } \\
\hline Própria & 45 & $90,0 \%$ \\
\hline Alugada & 5 & $10,0 \%$ \\
\hline Faz uso de medicação & 31 & $62,0 \%$ \\
\hline Realiza atividade física & 31 & $62,0 \%$ \\
\hline \multicolumn{3}{|l|}{ Autopercepção de saúde } \\
\hline Ótima & 9 & $18,0 \%$ \\
\hline Boa & 28 & $56,0 \%$ \\
\hline Regular & 12 & $24,0 \%$ \\
\hline Ruim & 1 & $2,0 \%$ \\
\hline Histerectomizada & 11 & $22,0 \%$ \\
\hline Faz reposição hormonal & 10 & $20,0 \%$ \\
\hline
\end{tabular}

mentais como médios. O domínio de qualidade de vida que mais pontuou foi o físico, significando que as mulheres possuem boa energia, se locomovem sem dificuldades e estão satisfeitas com o sono. E o que menos pontuou foi o meio ambiente, considerando que não estão satisfeitas com o local em que vivem. 
Tabela 2 - Autoestima e autoimagem, avaliação da menopausa e qualidade de vida e de saúde mental da mulher.

\begin{tabular}{|c|c|}
\hline & Média \pm desvio padrão \\
\hline Autoestima e Autoimagem & $131,60 \pm 15,06$ \\
\hline Escala de Avaliação da Menopausa (MRS) & $12,18 \pm 8,54$ \\
\hline MRS_Somática & $4,78 \pm 3,31$ \\
\hline MRS_Psicológica & $4,86 \pm 4,32$ \\
\hline MRS_Urogenital & $2,58 \pm 2,56$ \\
\hline Questionário da Saúde Mental da Mulher (QSM) & $96,74 \pm 16,56$ \\
\hline QSM_Depressivos & $16,82 \pm 2,58$ \\
\hline QSM_Ansiosos & $11,62 \pm 3,37$ \\
\hline QSM_Somáticos & $18,58 \pm 5,27$ \\
\hline QSM_Memória & $7,46 \pm 2,18$ \\
\hline QSM_Vasomotor & $5,70 \pm 2,07$ \\
\hline QSM_Sexualidade & $7,04 \pm 1,59$ \\
\hline QSM_Sono & $7,54 \pm 2,21$ \\
\hline QSM_Menstrual & $13,18 \pm 2,51$ \\
\hline QSM_Atratividade & $4,20 \pm 1,65$ \\
\hline \multicolumn{2}{|l|}{ Escala de qualidade de vida WHOQOL-Bref } \\
\hline Domínio Físico & $69,00 \pm 16,61$ \\
\hline Domínio Psicológico & $63,25 \pm 16,35$ \\
\hline Domínio das relações sociais & $66,50 \pm 16,19$ \\
\hline Domínio do meio ambiente & $62,25 \pm 11,56$ \\
\hline Qualidade de vida geral & $17,87 \pm 4,37$ \\
\hline Satisfação com a saúde & $15,87 \pm 5,53$ \\
\hline
\end{tabular}

Tabela 3 - Correlação entre a escala de Avaliação da Menopausa com as escalas de autoestima e autoimagem, questionário da saúde mental da mulher e qualidade de vida.

\begin{tabular}{|c|c|c|c|c|}
\hline & MRS & MRS Somática & MRS Psicológica & MRS Urogenital \\
\hline Autoestima Autoimagem & $-0,535^{* *}$ & $-0,398^{\star *}$ & $-0,464^{\star *}$ & $-0,368^{* *}$ \\
\hline QSM & $-0,726^{* *}$ & $-0,744^{\star *}$ & $-0,588^{\star *}$ & $-0,243$ \\
\hline Depressivos & $-0,246$ & $-0,366^{* *}$ & $-0,313^{*}$ & 0,257 \\
\hline Ansiosos & $-0,647^{* *}$ & $-0,548^{* *}$ & $-0,567^{* *}$ & $-0,229$ \\
\hline Somáticos & $-0,600^{\star *}$ & $-0,655^{\star *}$ & $-0,437^{\star *}$ & $-0,302^{*}$ \\
\hline Memória & $-0,592^{* *}$ & $-0,489^{* *}$ & $-0,541^{* *}$ & $-0,255$ \\
\hline Vasomotor & $-0,410^{* *}$ & $-0,472^{\star *}$ & $-0,322^{*}$ & $-0,173$ \\
\hline Sexualidade & $-0,396^{* *}$ & $-0,214$ & $-0,246$ & $-0,564^{* *}$ \\
\hline Sono & $-0,727^{\star *}$ & $-0,806^{* *}$ & $-0,607^{* *}$ & $-0,229$ \\
\hline Menstrual & $-0,571^{\star *}$ & $-0,563^{* *}$ & $-0,516^{* *}$ & $-0,267$ \\
\hline Atratividade & $0,347^{*}$ & $0,362^{\star *}$ & 0,232 & 0,063 \\
\hline \multicolumn{5}{|l|}{ WHOQOL-Bref } \\
\hline Domínio Físico &,$- 0637^{\star *}$ & $-0,671^{* *}$ & $-0,466^{* *}$ & $-0,311^{*}$ \\
\hline Domínio Psicológico & $-0,715^{* *}$ & $-0,620^{* *}$ & $-0,662^{* *}$ & $-0,327^{*}$ \\
\hline Domínio Relações sociais & $-0,360^{*}$ & $-0,261$ & $-0,372^{\star *}$ & $-0,233$ \\
\hline Domínio meio ambiente & $-0,502^{* *}$ & $-0,350^{*}$ & $-0,424^{\star *}$ & $-0,338^{*}$ \\
\hline
\end{tabular}


A avaliação por meio de correlação (Tabela 3) mostrou uma relação negativa e moderada entre o escore total da escala de Avaliação da Menopausa com a escala de autoestima e autoimagem $(r=-0,535 ; p<0,001)$ e com os sintomas do questionário de saúde mental da mulher, que são os ansiosos ( $\mathrm{r}=-0,647 ; \mathrm{p}<0,001)$, somáticos $(\mathrm{r}=-0,600 ; \mathrm{p}<0,001)$, memória $(\mathrm{r}=-0,592 ; \mathrm{p}<0,001)$, menstrual $(\mathrm{r}=-0,571 ; \mathrm{p}<0,001)$ e com os domínios físico $(\mathrm{r}=-0,637 ; \mathrm{p}<0,001)$ e meio ambiente $(\mathrm{r}=-0,502$; $\mathrm{p}<0,001)$ da escala de qualidade de vida. Também houve correlação negativa e moderada entre as subdivisões da escala de Avaliação da Menopausa: os sintomas somáticos com os sintomas do questionário de saúde mental da mulher, que são os ansiosos $(\mathrm{r}=-0,548 ; \mathrm{p}<0,001)$, somáticos $(\mathrm{r}=-0,655 ; \mathrm{p}<0,001)$, memória $(r=-0,489 ; \mathrm{p}<0,001)$, vasomotor $(\mathrm{r}=-0,472$; $\mathrm{p}<0,001)$, menstrual $(\mathrm{r}=-0,563 ; \mathrm{p}<0,001)$, domínios de QV físico $(r=-0,671 ; p<0,001)$ e psicológico $(r=-0,620$; $\mathrm{p}<0,001)$; os sintomas psicológicos com autoestima e autoimagem $(r=-0,464 ; \mathrm{p}<0,001)$, QSM total $(r=-$ $0,588 ; \mathrm{p}<0,001)$, sintomas ansiosos $(\mathrm{r}=-0,567 ; \mathrm{p}<0,001)$, somáticos $(r=-0,437 ; p<0,001)$ e memória da escala QSM ( $\mathrm{r}=-0,541 ; \mathrm{p}<0,001)$, sono $(\mathrm{r}=-0,607 ; \mathrm{p}<0,001)$, menstrual $(r=-0,516 ; p<0,001)$, domínio físico $(r=-$ 0,466; $\mathrm{p}<0,001)$, psicológico $(\mathrm{r}=-0,662 ; \mathrm{p}<0,001)$ e meio ambiente $(\mathrm{r}=-0,424 ; \mathrm{p}<0,001)$; e os sintomas urogenitais com a sexualidade do questionário QSM $(r=-0,564 ; p<0,001)$. Foi possível verificar relações negativas e fortes entre a pontuação total da escala de Avaliação da Menopausa e com a pontuação total da QSM ( $\mathrm{r}=-0,726 ; \mathrm{p}<0,001)$, sintomas de sono da QSM $(\mathrm{r}=-0,727 ; \mathrm{p}<0,001)$ e o domínio de QV psicológico ( $\mathrm{r}=-0,715 ; \mathrm{p}<0,001)$, além dos sintomas somáticos da escala de Avaliação da Menopausa com o valor total da QSM $(r=-0,744 ; p<0,001)$ e sono $(r=-0,806 ; p<0,001)$. As outras correlações existentes na tabela foram fracas.

\section{DISCUSSÃO}

Em relação aos dados socioeconômicos, a maioria das mulheres eram casadas, com renda de um a quatro salários-mínimos e com uma média de escolaridade de 9,48 \pm 3,92 anos de estudo. A principal patologia crônica autorreferida pelas mulheres foi a hipertensão arterial. Em um estudo semelhante sobre a saúde da mulher, a amostra foi composta por mulheres de 50 a 79 anos, na pósmenopausa, com alto percentual de gordura corporal, sedentárias, hipertensas e atendidas na Unidade de Saúde da Família em um município do Estado de São Paulo1 $^{1}$. Para outros pesquisadores ${ }^{12}$, no município de Porto Alegre observou-se uma taxa considerável de doenças crônicas não transmissíveis em mulheres $(81,3 \%)$, sendo a hipertensão arterial sistêmica a de maior prevalência $(70,8 \%)$.

No estudo em questão, a média de idade de início da menopausa foi de 47,16 $\pm 4,95$ anos. A maioria das mulheres avaliaram como boa sua autopercepção de saúde, 20,0\% faziam uso de reposição hormonal, $22 \%$ fizeram histerectomia, a maioria fazia uso de medicação e não praticava atividade física. Em uma pesquisa semelhante ${ }^{13}$, com 456 mulheres na pósmenopausa, a média de idade foi de 58,7 anos e a do início da menopausa de 48 anos, a maioria era casada, tinha uma média de escolaridade de 8 anos e a autopercepção de saúde de grande parte foi boa. Em outro estudo ${ }^{14}$, o perfil e intensidade dos sintomas de 56 mulheres no climatério foi com média de idade em torno de 51,1 $\pm 5,47$ anos, a maioria eram casada, dona de casa, não fazia reposição hormonal e 25\% fizeram histerectomia, resultado semelhante com os achados deste estudo.

Observou-se que houve uma insatisfação com a autoestima e autoimagem dessas mulheres. $\mathrm{Na}$ escala de qualidade de vida houve uma pontuação mais baixa nos domínios meio ambiente, que se refere ao ambiente físico, recreação e cuidados com a saúde e no domínio psicológico, demonstrando sentimentos negativos, insatisfação com a autoestima, imagem corporal e aparência ${ }^{15}$. Além disso, pontuaram sintomas moderados e médios nas escalas de avaliação da menopausa e saúde mental da mulher, demonstrando que essas mulheres 
não estão satisfeitas com os sintomas que sentem nessa fase da vida. Um achado importante foi que a maioria era de mulheres casadas, ou seja, que tinha seus companheiros, tinha uma vida conjugal, mas que refletia insatisfação em diversos aspectos relacionados ao corpo, saúde mental e qualidade de vida. Os sintomas, sinais físicos e subjetivos, podem prejudicar o bem-estar, conforto e qualidade de vida das mulheres nessa fase de pós-menopausa ${ }^{16}$.

Com relação à avaliação da autoestima e autoimagem das mulheres, a média do escore total foi de 131,60 $\pm 15,06$, considerado baixo pela escala avaliada, resultado este que se assemelha à pesquisa realizada com 47 mulheres idosas em São Luiz, Estado do Maranhão, que utilizou a mesma escala, em que grande parte das idosas analisadas mostrou uma média de 150 pontos $^{17}$. Esses dados diferem de outro estudo realizado na cidade de São Paulo, que avaliou a autoestima e cuidados pessoais em mulheres de 60 a 75 anos, apresentando como resultado uma autoestima elevada ${ }^{18}$.

Na escala de avaliação da menopausa (MRS), a média do escore total foi de 12,18 $\pm 8,54$, encaixando-se na classificação de sintomas moderados. A subescala de sintomas psicológicos teve uma média de 4,86 $\pm 4,32$, constatando nas mulheres avaliadas poucos sintomas de ansiedade, irritabilidade, ânimo depressivo e esgotamento físico e mental. A subescala somática obteve média de 4,78 $\pm 3,31$ relacionando com problemas de sono, falta de ar, calores, suores, mal-estar do coração, problemas musculares e nas articulações, que podem ter advindo da pósmenopausa. A terceira subescala, urogenital, teve média de 2,58 $\pm 2,56$. Dentre os sintomas avaliados estão ressecamento vaginal, problemas sexuais e problemas de bexiga. Pesquisadores ${ }^{19}$ revelam que $70,5 \%$ das mulheres climatéricas entre 40 e 65 anos com a MRS, apresentou, no domínio das questões psicológicas, avaliação de sintomas como severos, sendo que os que mais se repetiram foram os de origem depressiva; e, em 26,5\% delas a exaustão física predominou. Em relação ao domínio urogenital, grande parte (64\%) descreveu sintomas severos, em especial à ausência de desejo sexual e desprazer nos vínculos afetivos. Os sintomas de ressecamento vaginal e problemas urinários também tiveram relevância. No domínio somático, $71 \%$ das avaliadas categorizaram os sintomas de moderados a severos.

Além disso, estudos comprovam que, neste período, as mulheres estão mais predispostas à ansiedade e depressão, devidoà redução da liberação de endorfinas cerebrais decorrentes das diminuições hormonais ${ }^{20}$. Nesse período, as mulheres podem apresentar alterações relacionadas a fatores psicológicos, culturais, emocionais e sociais, apresentando maior ou menor sintomatologia. Através dessas mudanças é possível observar modificações em seus corpos, com manifestações distintas, estranhas, confusas e muitas vezes interiorizadas, expressando através de ondas de calor, suores "frios", insônia, tristeza, instabilidade emocional, modificações nos hábitos sexuais, na pele e na distribuição da gordura corporal ${ }^{21}$.

No Questionário de Saúde Mental da Mulher observou-se um escore geral de 96,74 $\pm 16,56$, considerado como uma pontuação média de sintomas físicos e psicológicos. Os domínios que tiveram maior pontuação, ou seja, positivos, foram o menstrual $(3,29) 13,18 \pm 2,51$, seguido pelo ansioso $(2,90) 11,62 \pm$ 3,37 e vasomotor $(2,85) 5,70 \pm 2,07$, podendo concluir que as mulheres analisadas não apresentaram muitos sintomas climatéricos nesta escala. Os domínios que menos pontuaram foram o depressivo $(2,40)$ 16,82 $\pm 2,58$, seguido por sexualidade $(2,34) 7,04 \pm 1,59 \mathrm{e}$ atratividade $(1,40) 4,20 \pm 1,65$. Pode-se considerar que estas mulheres não estão satisfeitas com seu corpo, com suas relações íntimas e possuem sentimentos negativos.

Em um estudo semelhante ${ }^{22}$, realizado com 250 mulheres na pós-menopausa, que realizam, ou não, reposição hormonal, com idades entre 45 e 70 anos, atendidas em uma Unidade Básica de Saúde (UBS), constatou-se que mulheres que fazem $\mathrm{TH}$ (terapia hormonal) tiveram um escore menor em 
relação a sintomas vasomotores, atratividade e problemas do sono. Quanto aos sintomas somáticos, depressivos, sexuais e problemas menstruais não foi identificada mudança relevante. Em uma pesquisa com o objetivo de avaliar mulheres no climatério em relação à qualidade de vida, as participantes foram separadas em duas turmas: 49 com tempo menor que 5 anos de pós-menopausa, e 61 com tempo maior que 5 anos e menor que 15 . Na avaliação do Questionário de Saúde da Mulher achou-se um grau mediador de QV, não existindo distinção entre as duas turmas. Em relação aos domínios de memória/ concentração, atratividade e sintomas vasomotores, foram verificadas consideráveis diferenças, dispondo de maiores médias de escores as mulheres da pósmenopausa recente ${ }^{23}$.

A redução progressiva dos níveis hormonais, vinculada ao processo de envelhecimento, favorece a falta de interesse levando em conta a diminuição da frequência de atividade sexual. Porém, fatores não hormonais estão "associados" com a redução da libido e função sexual de mulheres nesta etapa da vida como o estado emocional, qualidade de relacionamento e ambiente ${ }^{20}$.

Nos dados que avaliaram a qualidade de vida, através da aplicação da escala WHOQOL-BREF, concluiuse que as participantes apresentaram uma maior satisfação no domínio físico referente à mobilidade, dependência de medicações e capacidade de trabalho ${ }^{15}$; e, menor satisfação com o meio ambiente. Outros achados com a escala WHOQOL-Bref evidenciaram melhor satisfação com o domínio físico e menor com o psicológico ${ }^{24}$. Os resultados vêm de encontro às correlações visualizadas entre as escalas (Tabela 3), que demonstram que quanto maior os sintomas da escala de avaliação da menopausa, menor a autoestima e autoimagem, qualidade de vida e saúde da mulher.

Diante dos resultados encontrados observa-se o quanto uma intervenção educacional com mulheres climatéricas seria uma importante ferramenta de promoção de QV. A educação em saúde para mulheres representa um dos recursos com maior capacidade de gerar impactos positivos para a saúde feminina, contribuindo para maior cuidado pessoal, mudança de crenças negativas a respeito do envelhecimento feminino e esclarecimento de dúvidas sobre o climatério e a menopausa. Também pode ser definida como uma combinação de experiências de aprendizagem planejadas para ajudar indivíduos e comunidades na melhoria de sua saúde, aumentar seu conhecimento e influenciar suas atitudes ${ }^{25,26}$.

Existem ações de educação em saúde que são desenvolvidas no coletivo, como rodas de conversa que se pautam na reflexão crítica em relação às problemáticas e ações de melhoria das condições de saúde, evidenciando a autonomia das mulheres. Oficinas de educação em saúde são espaços de construção de conceitos que facilitam a expressão individual e coletiva de necessidades e expectativas, espaços de diálogo buscando contribuir socialmente e melhorar a qualidade de vida da mulher no climatério ${ }^{27}$.

\section{CONCLUSÕES}

A partir deste estudo, que teve como objetivo avaliar a autoestima, autoimagem, qualidade de vida e de saúde de mulheres na pós-menopausa conclui-se que as mulheres apresentaram baixa autoestima e autoimagem, revelando não estarem satisfeitas com este aspecto corporal. A avaliação geral da menopausa indica que as mulheres apresentam sintomas moderados. A subescala de sintomas somáticos se mostraram leves, ou seja, menos relatados (problemas de sono, falta de ar, calores, suores, mal-estar do coração, problemas musculares e nas articulações); já na de psicológicos (ansiedade, irritabilidade, ânimo depressivo, e esgotamento físico e mental) e urogenitais (ressecamento vaginal, problemas sexuais, e problemas de bexiga) os sintomas são moderados, ou seja, bastante relatados. 
No questionário de saúde mental da mulher os sintomas físicos e psicológicos foram classificados como médios, pontuando mais os sintomas depressivos, ansiosos, somáticos e menstruais. Entretanto, na escala de qualidade de vida o domínio que teve maior satisfação foi o físico; e, menor foi o meio ambiente, demonstrando que essas mulheres estão satisfeitas com suas atividades do dia a dia e mobilidade, porém não estão muito satisfeitas com o lugar em que vivem, com os recursos financeiros que possuem e meios de transporte utilizados.

Por fim, este estudo apresenta algumas limitações que devem ser consideradas, como se basear em uma amostra composta por conveniência e os resultados não podem ser generalizados por se tratar de uma amostra específica da população de mulheres na pós-menopausa. Neste sentido, espera-se, em estudos futuros, avaliar mais mulheres em diferentes contextos para se obter dados mais amplos. Além disso, verificar a importância de ações de educação em saúde com mulheres nessa fase da vida.

\section{REFERÊNCIAS}

1. Ferreira LL, Cochito TC, Caíres FD, Marcondes LP, Saad PCB. Capacidade funcional de idosos institucionalizados com e sem doença de Alzheimer. Revista Brasileira de Geriatria e Gerontologia. 2014; 17(3):567-573. doi:10.1590/1809-9823.201

2. Instituto Brasileiro de Geografia e Estatística. Dados sobre População do Brasil, PNAD (Pesquisa Nacional por Amostra de Domicílios), quantidade de homens e mulheres, 2019. https://educa.ibge.gov.br/jovens/conheca-o-brasil/ populacao/18320-quantidade-de-homens-e-mulheres.html

3. Almeida AV, Mafra SCT, Silva EP, Kanso S. A Feminização da Velhice: em foco as características socioeconômicas, pessoais e familiares das idosas e o risco social. Textos \& Contextos. 2015;14(1):115-131. doi:10.15448/1677-95091.2015.1.19830

4. Tomás CC, Pimenta F, Costa PA, Maroco JA, Leal I. Representações e consequências percebidas da menopausa e andropausa: resultados preliminares do EVISA. Psicologia, Saúde \& Doenças. 2018; 19(1): 87-93.

5. Brasil. Protocolos da Atenção Básica: Saúde das Mulheres. Ministério da Saúde, Instituto Sírio-Libanês de Ensino e Pesquisa. Brasília: Ministério da Saúde, 2016.

6. Cobo CMS. La imagem corporal enlosancianos: Estudiodescriptivo. Gerokomos: Revista de la Sociedade Española de Enfermería Geriátrica y Gerontológica. 2012; 23(1):15-18. doi:10.4321/S1134-928X2012000100003

7. Fleck MPA, Louzada S, Xavier M, Chachamovich E, Vieira G, Santos L, et al. Aplicação da versão em português do instrumento abreviado de avaliação de qualidade de vida WHOQOL-BREF. Revista de Saúde Pública, 2000; 34(2):178-183.

8. Stobäus CD. Desempenho e auto-estima em jogadores profissionais e amadores de futebol: análise de uma realidade e implicações educacionais. (Dissertação Mestrado em Educação) Universidade Federal do Rio Grande do Sul, Porto Alegre, Rio Grande do Sul, 1983.

9. Heinemann LA, Potthoff P, Schneider HP. International versions of the menopause rating scale (MRS). Health and quality of life outcomes. 2003; 1(1):1-28. doi:10.1186/14777525-1-28

10. Dias R, Ramos CC, Kerr-Corrêa F, Trinca A, Cerqueira ATAR, Dalben I et al. Adaptação para o português do questionário de autoavaliação de percepção de saúde física e mental da mulher de meia-idade - Questionário da Saúde da Mulher. Revista de Psiquiatria Clínica. 2002; 29(4):181189.

11. Chagas EFB, Bonfim MR, Brondino NCM, Monteiro HL. Exercício físico e fatores de risco cardiovasculares em mulheres obesas na pós-menopausa. Rev Bras Med Esporte. 2015;21(1):65-69. doi: 10.1590/1517-86922015210101834

12. Silva AR, Sgnaolin V, Nogueira EL, Loureiro F, Engroff $\mathrm{P}$, Gomes I. Doenças crônicas não transmissíveis e fatores sociodemográficos associados a sintomas de depressão em idosos. Jornal Brasileiro de Psiquiatria. 2017;66(1):45-51. doi:10.1590/0047-2085000000149

13. Gravena AAF, Rocha SC, Romeiro TC, Agnolo CMD, Gil LM, Carvalho MD et al. Sintomas climatéricos e estado nutricional de mulheres na pós-menopausa usuárias e não usuárias de terapia hormonal. (Dissertação - pós-graduação em Enfermagem) Universidade Estadual de Maringá, 2013. 
14. Peixoto LN, Araujo MFS, Egydio CA, Ribeiro FE, Fregonesi CEPT, Carmo EM. Perfil e intensidade de sintomas de mulheres no climatério avaliadas em unidades básicas de saúde de Presidente Prudente. Colloq Vitae. 2015;7(1):85-93. Recuperado de http://journal.unoeste.br/ index.php/cv/article/view/1267

15. Gorestein C, Wang YP, Hungerbühler I. Instrumentos de avaliação em saúde mental. Artmed, Porto Alegre, 2016.

16. Santos LS, Lemos MD, Zorzim VI. Mulheres menopausadas e suas crenças sobre esta fase da vida. Braz. J. of Develop. 2019; 5(6):6892-6906. doi:10.34117/bjdv5n6-179

17. Costa ECS, Andrade GMM, Jinkings YF, Corrêa RGCF, Aquino DMC. (2011). Avaliação da autoestima em idosos de um Centro Integral à Saúde do Idoso - São Luís - MA. Revista de Pesquisa em Saúde. 2011; 12(1):9-13.

18. Duridan A, Santos DF, Gatti AL. Autoestima e cuidados pessoais em mulheres de 60 a 75 anos. Aletheia. 2014; 3(1):174-187. Disponível em: http://pepsic. bvsalud.org/scielo.php?script=sci_arttext\&pid=S141303942014000100013\&lng=pt.

19. Gallon CW, Wender COM. Estado nutricional e qualidade de vida da mulher climatérica. Revista Brasileira Ginecologia e Obstetrícia. 2012; 34(4):175-183.

20. Cabral PUL, Canário ACC, Spyrides MHC, Uchôa SAC, Eleutério Júnior J et al. Influência dos sintomas climatéricos sobre a função sexual de mulheres de meia-idade. Revista Brasileira de Ginecologia e Obstetrícia. 2012; 34(7):329-334. https://doi.org/10.1590/S0100-72032012000700007

21. Conte FA, Franz LBB. Estado nutricional e de saúde em mulheres pós-menopausa. Saúde (Santa Maria). 2015; 41(1):85-92. doi.org/10.5902/2236583412837

22. Martins MAD, Nahas EAP, Nahas-Neto J, Uemura G, Buttros DDAB, Traiman P. Qualidade de vida em mulheres na pós-menopausa, usuárias e não usuárias de terapia hormonal. Revista Brasileira de Ginecologia e Obstetrícia. 2009; 31(4):196-202. doi:10.1590/S0100-72032009000400007

23. Melo Filho SSA. Cefaleia e qualidade de vida em mulheres em pós-menopausa recente e tardia. (Dissertação de mestrado - Universidade Federal de Pernambuco), 2012.

24. Irigary TQ, Schneider RH. Dimensões de personalidade, qualidade de vida e depressão em idosas. Psicologia em Estudo, Maringá. 2009 ;14(4):759-766.

25. Garcia FAR, Freund KM, Berlin M, Digre KB, Dudley DJ, Fife RS, et al. Progress and priorities in the health of women and girls: a decade of advances and challenges J Womens Health, 2010; 19:671-680.

26. Sousa JDL, Zveiter M, Almeida VLMD, Menezes HFD, Mara G, Alves R. Educação em saúde como ferramenta à mulher no climatério: subsídios para o cuidado de enfermagem. Rev Pesq.: Cuid Fundam., 2012; 4:2616-2622.

27. Baraibar D, Ferreira LP, Fernandes MTC, Dellanhese APF. Práticas de educação em saúde para promoção da qualidade de vida de mulheres climatéricas. Saúde Coletiva, 2020;10(56):3176-3180. 